\title{
Optimization of Earthwork Allocation Path as Vehicle Route Problem Based on Genetic Algorithm
}

\author{
Naifu Deng ${ }^{1, *}$, Xuyang $\mathrm{Li}^{1}$ and Yanmin $\mathrm{Su}^{1}$ \\ ${ }^{1}$ CCCC Tunnel Engineering Company Limited, Beijing 10000, China
}

\begin{abstract}
In civil engineering, earthwork, prior to the construction of most engineering projects, is a lengthy and time-consuming work involving iterative processes. The cost of many AEC (Architecture, Engineering and Construction) projects is highly dependent on the efficiency of earthworks (e.g. road, embankment, railway and slope engineering). Therefore, designing proper earthwork planning is of importance. This paper simplifies the earthwork allocation problem to Vehicle Route Problem (VRP) which is commonly discussed in the field of transportation and logistics. An optimization model for the earthwork allocation path based on the modified Genetic Algorithm with a self-adaptive mechanism is developed to work out the global optimal hauling path for earthwork. The research results also instruct the initial topographic shaping of the Winter Olympic Skiing Courses Project. Furthermore, this optimization model is highly compatible with other evolutionary algorithms due to its flexibility, therefore, further improvement in this model is feasible and practical.
\end{abstract}

\section{Introduction}

Earthwork allocation is a huge and repetitive work required by a great majority of AEC (Architecture, Engineering and Construction) projects [1]. Earthworks, or earthwork allocation, refer to the process of levelling or shaping the ground surface of target areas by moving or treating the geological materials that comprise the target areas, and the allocation of this geological material usually involves excavating, loading, hauling, unloading, compacting where usually implemented in different areas, sometimes it may also include some intermediate steps, such as, material mixture or treatment [2]. Due to the complexity of earthworks activities, it always incurs a large amount of project cost and usually takes up 50\% of the overall cost. Among the steps mentioned above, hauling the geotechnical materials from one location to another costs the highest expenditure in most cases. Therefore, finding an efficient and technical method to properly program earthworks path can obtain many benefits because earthwork allocation is typically an iterative process that can be utilized continually [1]

Earthwork allocation is essentially an extended application of Vehicle Route Problem (VRP). VRP is a combinatorial dynamic program problem seeking to serve a certain number of customers with different demands of goods by a fleet of vehicles under certain constraints, such as delivery time or load capacity of nominated vehicles, it mainly refers to a class of problems in which an optimal route for a fleet of vehicles based on one or several depots can be determined for a number of customers dispersed geographically [3]. Fig 1 represents a possible solution to a single-depot VRP. Vehicle Route Problem, proposed by
Dantzig and Ramser in 1959 [3], is a very important problem in the field of transportation and logistics, the problem from the date it arose has attracted great attention from experts and researchers, and has contributed to the development of Transportation, aviation, navigation, communications, electric power, computer and other fields in certain aspects. However, the VRP is an NP-hard problem, which cannot be completed in polynomial time. In other words, it is impossible to find the exact solution of the problem with the current knowledge level, or it will take a long time to find the exact solution, especially when the problem is in large scale, it is often impossible to find the optimal solution. Therefore, recent research has shifted from the precise algorithm to find the optimal solution to the heuristic algorithm to find a better solution.

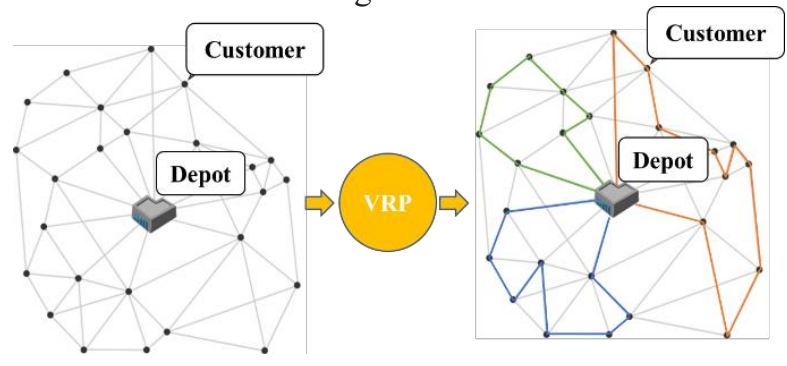

Fig 1. One possible solution for single-depot VRP.

The commonly used heuristic algorithms are Genetic Algorithm (GA)[4], Ant Colony Algorithm (ACA)[5], Particle Swarm Optimization Algorithm (PSOA)[6], Simulated Annealing Algorithm (SAA)[7], Tabu Search Algorithm (TSA)[8] and so on. In this paper, GA is adopted to study the earthwork allocation path problem, because GA appeared earlier, and it is also one of the

*Naifu Deng: nden8417@163.com 
earliest algorithms to be applied to the field of transportation. Additionally, researches show that GA is very suitable for solving NP-hard problems with multiple variables, multiple parameters, multiple targets and poor connectivity in multiple regions. GA can also record multiple solutions at the same time, and the process of simultaneous optimization for multiple solutions is also suitable for solving multi-objective optimization problems. Moreover, GA inherits higher universality and compatibility which enable further room for improvement combining with other heuristic algorithms.

Therefore, this paper focuses on the Vehicle Route Problem to refine the earth allocation problem based on the Genetic Algorithm. In reality, earth allocation may include multiple vehicle replenishment centres (depots), multiple excavation areas (distribution centres) and multiple filling areas (customers) distributed geographically in a certain range of areas. Each vehicle sent out from arbitrary replenishment centres needs an initial start-up cost and each vehicle must return to any replenishment centre to refuel before it reaches its mileage limit. In addition, the fuel consumption of vehicles in empty and full load conditions may also vary. Furthermore, there is also an upper limit to the amount of earthwork produced by each excavation areas, thus, an excavation area cannot provide unlimited earthwork to its nearest filling areas, these filling areas must select another possible excavation area to provide earthwork when the earthwork from their closest excavation areas is exhausted. Therefore, this paper aims to optimize the number of dispatched vehicles and overall delivery cost over shipment distance considering the constraints proposed above.

\section{Model development}

\subsection{Problem description}

GPS technique is used to acquire the real location information (position nodes) of each replenishment centre (depot), excavation area (distribution centre) and filling area (customer), where-after, a transportation network can be built by linking all available position nodes together based upon real terrain. Supposed there are $\Pi$ nodes in the transportation network, nodes $1,2, \ldots, m$ are for replenishment centre, $m+1, \ldots, m+n$ are for excavation areas and $m+n+1, \ldots, m+n+s$ are for filling areas where $\Pi=m+n+s$.

There are $\beta_{i}$ vehicles in $i t h(i=1, \ldots, m)$ replenishment centre, and the earth volume dredged in $j t h(j=m+1, \ldots, m+n)$ excavation area requires $\gamma_{j}$ fully loaded vehicles to haul while the earthwork needed in $k t h(k=m+n+s, \ldots, m+n+s)$ filling area demands $\rho_{k}$ fully loaded vehicles to unload. Meanwhile, the earthwork from all excavation area should be slightly larger than that needed in all filling areas, that is $\sum_{j=m+1}^{j=m+n} \gamma_{j}>\sum_{k=m+n+1}^{k=m+n+s} \rho_{k}$.

Empty-loaded vehicle starts from their replenishment centres to any excavation area, and then head to any filling area after fully loaded with earthwork. Where-after, it can either return to one of the replenishment centres to refuel (this is called one-way vehicle) or continue its earthwork hauling between excavation areas and filling areas (this is called multi-way vehicle), in this paper, two-way vehicle problem is mainly addressed. It is also required that each replenishment centre send the same number of vehicles as they return to it (because each centre's vehicles may belong to a different company in reality).

In this paper, the maximum mileage obtained by oneway vehicles is set as the mileage limit of all vehicles. On the basis of not exceeding the mileage limit, each dispatched vehicle can return to the excavation areas with earthwork stock for multiple deliveries. Additionally, all vehicles have the same start-up cost. The purpose of this paper is to find out an optimal delivery scheme enabling the fewest dispatched vehicles and minimum shipment costs. Therefore, based on the research model by Sun Rui[9], this paper introduces an self-adaptive mechanism to avoid premature convergence and improve the overall performance to find out the global optimal solution.

\subsection{Objective model and constraints}

The implication of variables:

$\alpha_{i j}$ : the number of dispatched vehicles from node $i$ to $j$ ( $\alpha_{i j}$ is a positive integer);

$\beta_{i}$ : the overall number of vehicles in ith $(i=1, \ldots, m)$ replenishment centre $\left(\beta_{i} \geq \alpha_{i j} \geq 0\right)$;

$\gamma_{j}$ : the number of fully loaded vehicles needed to haul all earthwork stock in $j t h(j=m+1, \ldots, m+n)$ excavation area;

$\rho_{k}$ : the number of fully loaded vehicles needed to satisfy the demand in $k t h(k=m+n+1, \ldots, m+n+$ $s)$ filling area;

$\mu_{0}$ : the unit mileage charge of each empty-loaded vehicle;

$\mu_{1}$ : the unit mileage charge of each fully loaded vehicle $\left(\mu_{1} \geq \mu_{0}>0\right)$;

$\eta_{i j}$ : the distance between node $i$ and $j$;

$\tau$ : the mileage limit for each vehicle;

$\omega$ : the start-up cost for each vehicle;

$\tau_{i}^{S}$ : the accumulative driving distance for $s t h$ vehicle from ith $(i=1, \ldots, m)$ replenishment centre.

Thus, the objective model can be described as follows:

$$
\begin{gathered}
\min Z=\sum_{i=1}^{m} \sum_{j=m+1}^{m+n}\left(\mu_{0} \eta_{i j}+\omega\right) \alpha_{i j}+ \\
\sum_{j=m+1}^{m+n} \sum_{k=m+n+1}^{m+n+s} \mu_{1} \eta_{j k} \alpha_{j k}+ \\
\sum_{k=m+n+1}^{m+n+s} \sum_{j=m+1}^{m+n} \mu_{0} \eta_{k j} \alpha_{k j}+ \\
\sum_{k=m+n+1}^{m+n+s} \sum_{i=1}^{m} \mu_{0} \eta_{k i} \alpha_{k i}
\end{gathered}
$$

s.t.

$$
\begin{gathered}
\sum_{i=1}^{m} \sum_{j=m+1}^{m+n} \alpha_{i j} \leq \sum_{j=m+1}^{m+n} \beta_{j} \\
\sum_{j=m+1}^{m+n} \sum_{k=m+n+1}^{m+n+s} \alpha_{j k} \leq \sum_{j=m+1}^{m+n} \gamma_{j} \\
\sum_{j=m+1}^{m+n} \sum_{k=m+n+1}^{m+n+s} \alpha_{j k}=\sum_{k=m+n+1}^{m+n+s} \rho_{k} \\
\tau_{i}^{s} \leq \tau,\left(i=1, \ldots, m ; s=1, \ldots, \beta_{i}\right) \\
\sum_{i=1}^{m} \sum_{j=m+1}^{m+n} \alpha_{i j}=\sum_{k=m+n+1}^{m+n+s} \sum_{i=1}^{m} \alpha_{k i}
\end{gathered}
$$




$$
\sum_{j=m+1}^{m+n} \gamma_{j}>\sum_{k=m+n+1}^{m+n+s} \rho_{k}
$$

Where:

Eq (1) is the objective function modified based on Sun's model[9], on the RHS of this equation, the first section is the sum of the total empty running cost and total start-up cost of all vehicles dispatched from the replenishment centres to the excavation areas; the second section is the overall mileage charge of each fully-loaded vehicle from replenishment centres to excavation areas; the third section is the sum of the total empty running cost from filling areas to excavation areas; the fourth section is the overall mileage charge of all used vehicles returning to the replenishment centres from the filling areas. Eq (2) represents that the dispatched vehicles from each replenishment centre must not exceed the number of vehicles it owns. Eq (3) shows that the volume of earthwork transported from each excavation area shall not exceed its own earthwork storage capacity. Eq (4) means that the number of vehicles arriving at each filling area is equal to its required number. Eq (5) implies that the shipment distance of each vehicle shall not exceed the mileage limit. Eq (6) stipulates that the number of vehicles dispatched and returned must be equal. Eq (7) indicates that the earth volume in all excavation areas should be slightly greater than that in all filling areas.

\subsection{Design of genetic algorithm}

The Genetic Algorithm is a heuristic algorithm based on "Survival of the Fittest" and "Natural Selection" hypothesis. Fig 2 demonstrates the process and mechanism of how randomly generated individuals compete with each other and create new generations with self-adaptive crossover and mutation strategies. In the final step, when the system converges to a steady solution, it means the optimal solution found.

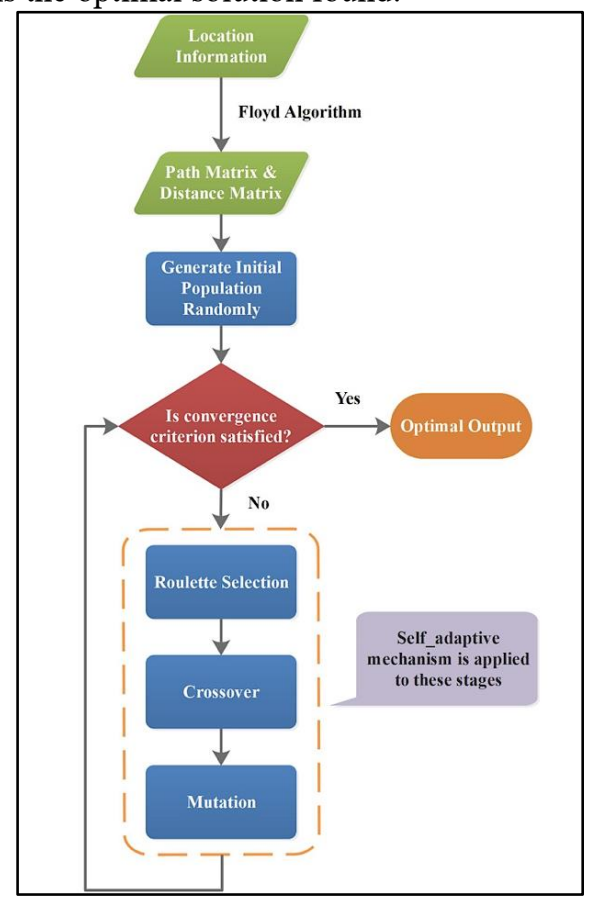

Fig 2. Flow Chart of Genetic Algorithm.

\subsubsection{Generation of the initial population}

Floyd Algorithm is adopted to determine the shortest distance between arbitrary point $i$ and $j$ in the original nodes network based on dynamic programming method[10]. The idea of Floyd Algorithm is to find a third point $k$ between point $i$ and $j$ competing the distance from $i$ to $j$ directly and from point $i$ to $j$ via $k$, if the latter distance is shorter, path $i \rightarrow k \rightarrow j$ will be updated as the shortest distance between point $i$ and $j$. Therefore, the recursive process of Floyd Algorithm can be expressed as[10]:

$$
d^{k}(i, j)=\min \left(d^{k-1}(i, j), d^{k}(i, k)+d^{k-1}(k, j)\right)
$$

Suppose that there are 100 units (chromosomes) in each generation and each chromosome has two-part, one is the feasible solution while the other is the fitness value of this feasible solution. Therefore, the information of a chromosome can be stored in a $1 \times 2$ Cell Array. Because $\rho=\rho_{m+n+1}+\cdots+\rho_{m+n+s}$ is the overall demands of all filling areas, the coding for the first element in the Array can be designed as a $c$ rows $\times 6$ columns matrix to store the information of a feasible solution. A One-way Vehicle feasible solution can be expressed as one row in the matrix, 6 elements in this row is placed in order as: 0 , The serial number of the departure replenishment centre, the serial number of the excavation area arrived at, the serial number of the filling area reached, the serial number of the returned replenishment centre, and the total mileage of the vehicle passing through the above nodes. A $x$-way Vehicle feasible solution can be stored in $x$ rows of matrix, take $x=2$ as an example, 6 elements in the 1st row stores: 1 , the serial number of the departure replenishment centre, the serial number of the excavation area arrived at, the serial number of the filling area reached, 0 and the total mileage of the vehicle passing through the above nodes; In the second row, 6 elements are stored successively: 2, the serial number of the first filling area arrived at, the serial number of the excavation area returned, the serial number of the filling area reached for the second time, the serial number of the replenishment centre returned, and the total mileage through the above nodes. The fitness value of each chromosome, stored in the second element of the Array, is the sum of the total mileage cost and the total start-up cost of all vehicles in the corresponding feasible solution.

\subsubsection{Roulette selection}

Roulette Selection is to determine some better individuals from the paternal chromosome according to their related fitness value, the smaller the fitness value is, the more possibility its corresponding chromosomes will be inherited. Additionally, Elitism Strategy is applied to the generated offspring after the above random operation preserving the best paternal genes by replacing the worst feasible solution of the offspring with the best feasible solution of the parent. 


\subsubsection{Crossover}

In this model, only the individual pairs of chromosomes that meet the following conditions can intersect: in two feasible solutions corresponding to two chromosomes, 1. they both contain one-way vehicle solutions; 2 . they have the same departure and returned replenishment centres in their one-way vehicle solutions; 3 . one of each of the vehicles satisfying 1 and 2 is selected, whose corresponding replenishment centres and the excavation areas corresponding are capable to interact (the replenishment centres and the excavation areas need to be different), then the two chromosomes can swap the replenishment centres and the excavation areas of the selected vehicles, and the overall mileage and fitness value are recalculated thereafter. Crossover is controlled by a self-adaptive mechanism as follow, $d$ is the number of optimal feasible solution and $N$ is the population size.

$$
\begin{gathered}
\text { Crossover Rate }=0.3 \times t_{1}, \\
t_{1}=\left(\begin{array}{c}
2.5, d \geq 0.6 \times N \\
1.5, d \geq 0.3 \times N \\
1, d<0.3 \times N
\end{array}\right)
\end{gathered}
$$

\subsubsection{Mutation}

This paper implements the following operations on the selected mutant individuals: modifying the third element (excavation areas) in the row where the first element of the feasible solution corresponding to the individuals is 0 and 1 as following operations: 1. replacing the excavation area of the row with another one still with earthwork capacity, which enable a shorter distance from the replenishment centre to the filling area via the selected excavation area. Therefore, it is necessary to make the amount of earthwork in the excavation areas slightly larger than the volume of earthwork in the filling areas when modelling, because it can provide the individual with space for variation; 2 . After operation 1 , the length of the path travelled by the vehicle and the corresponding fitness of the entire feasible solution is updated. The mutation is also controlled by a self-adaptive mechanism as below:

$$
\begin{aligned}
& \text { Mutation Rate }=0.01 \times t_{2}, \\
& t_{2}=\left(\begin{array}{ll}
50, & d \geq 0.6 \times N \\
20, & d \geq 0.3 \times N \\
15, & d<0.3 \times N
\end{array}\right)
\end{aligned}
$$

\subsubsection{Recursive optimization}

By repeating 2.3.2 to 2.3.4 and iteratively updating the chromosomal information of each generation of the population, a better solution can be found with the smallest fitness value.

\section{Case study}

The case study in this paper is based on the optimization problem of earthwork allocation of the G1 excavation skiing course and D2 filling skiing course of Beijing Winter Olympic Games skiing centre in 2020. The original transportation network is shown in Fig 3 and the initial conditions of this case study are listed in Table 1.

\begin{tabular}{|c|c|c|}
\hline & Node & Number of Vehicles \\
\hline \multirow{2}{*}{$\begin{array}{l}\text { Replenishment } \\
\text { Centres }\end{array}$} & 1 & 1200 \\
\hline & 2 & 1200 \\
\hline \multirow{3}{*}{$\begin{array}{l}\text { Excavation } \\
\text { centres }\end{array}$} & 3 & 835 \\
\hline & 4 & 785 \\
\hline & 5 & 732 \\
\hline \multirow{7}{*}{ Filling Centres } & 6 & 280 \\
\hline & 7 & 260 \\
\hline & 8 & 330 \\
\hline & 9 & 300 \\
\hline & 10 & 250 \\
\hline & 11 & 334 \\
\hline & 12 & 224 \\
\hline
\end{tabular}

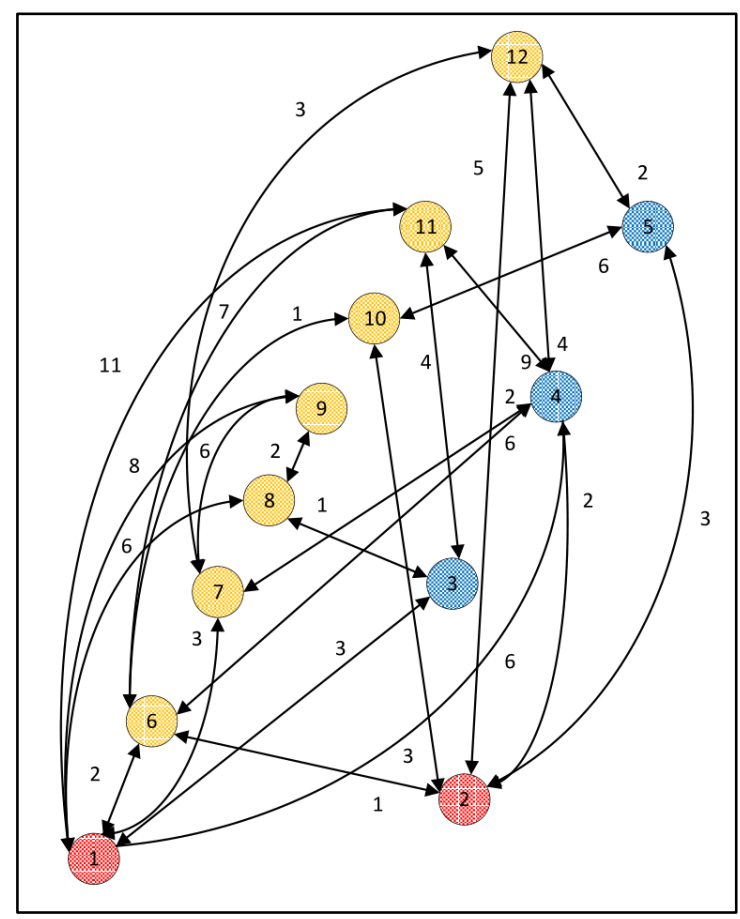

Fig 3. Original transportation network.

Table 1. Initial information for all nodes.

The overall demand of all filling areas is 1978, thus the final result is a $1978 \times 6$ matrix, each row represents the path information of one vehicle. Table 2 demonstrates the results of the optimal solution in the first 10 rows, from the first 10 deliveries, a total of 5 vehicles are dispatched for secondary deliveries and they all go through the same optimal route. 
Table 2. Optimal Solution of the First 10 Rows.

\begin{tabular}{|c|c|c|c|c|c|}
\hline 1 & 2 & 4 & 6 & 0 & 5 \\
\hline 2 & 6 & 3 & 9 & 2 & 17 \\
\hline 1 & 2 & 4 & 6 & 0 & 5 \\
\hline 2 & 6 & 3 & 9 & 2 & 17 \\
\hline 1 & 2 & 4 & 6 & 0 & 5 \\
\hline 2 & 6 & 3 & 9 & 2 & 17 \\
\hline 1 & 2 & 4 & 6 & 0 & 5 \\
\hline 2 & 6 & 3 & 9 & 2 & 17 \\
\hline
\end{tabular}

\begin{tabular}{|c|c|c|c|c|c|}
\hline 1 & 2 & 4 & 6 & 0 & 5 \\
\hline 2 & 6 & 3 & 9 & 2 & 17 \\
\hline
\end{tabular}

Therefore, the optimal path can be read assisted by table 3 generated from Floyd Algorithm: $2 \rightarrow 4 \rightarrow 2 \rightarrow 6 \rightarrow 1 \rightarrow 3 \rightarrow 8 \rightarrow 9 \rightarrow 8 \rightarrow 3 \rightarrow 1 \rightarrow 6 \rightarrow 2 \quad$ (for instance, from node 6 to 3 , node 1 can be read from Table 3 ), the total delivery distance is 22 unit-length. Meanwhile, Fig 4 is the iterative optimizing process, the iteration operated 500 times in total, however, the optimal value tended to be stable after 50 generations based on the advantages of the algorithm.

Table 3. Floyd path table.

\begin{tabular}{|c|c|c|c|c|c|c|c|c|c|c|c|c|c|}
\hline & Node & \multicolumn{12}{|c|}{ Destination } \\
\hline Node & & 1 & 2 & 3 & 4 & 5 & 6 & 7 & 8 & 9 & 10 & 11 & 12 \\
\hline \multirow{12}{*}{ Origin } & 1 & 1 & 6 & 3 & 6 & 6 & 6 & 7 & 3 & 3 & 6 & 3 & 7 \\
\hline & 2 & 6 & 2 & 6 & 4 & 5 & 6 & 4 & 6 & 6 & 6 & 6 & 12 \\
\hline & 3 & 1 & 1 & 3 & 1 & 1 & 1 & 1 & 8 & 8 & 1 & 11 & 1 \\
\hline & 4 & 2 & 2 & 2 & 4 & 2 & 2 & 7 & 2 & 7 & 2 & 11 & 12 \\
\hline & 5 & 2 & 2 & 2 & 2 & 5 & 2 & 12 & 2 & 12 & 2 & 2 & 12 \\
\hline & 6 & 1 & 2 & 1 & 2 & 2 & 6 & 1 & 1 & 1 & 10 & 11 & 2 \\
\hline & 7 & 1 & 4 & 1 & 4 & 12 & 1 & 7 & 1 & 9 & 1 & 1 & 12 \\
\hline & 8 & 3 & 3 & 3 & 3 & 3 & 3 & 3 & 8 & 9 & 3 & 3 & 3 \\
\hline & 9 & 8 & 8 & 8 & 7 & 7 & 8 & 7 & 8 & 9 & 8 & 8 & 7 \\
\hline & 10 & 6 & 6 & 6 & 6 & 6 & 6 & 6 & 6 & 6 & 10 & 6 & 6 \\
\hline & 11 & 3 & 6 & 3 & 4 & 6 & 6 & 3 & 3 & 3 & 6 & 11 & 4 \\
\hline & 12 & 7 & 2 & 7 & 4 & 5 & 2 & 7 & 7 & 7 & 2 & 4 & 12 \\
\hline
\end{tabular}

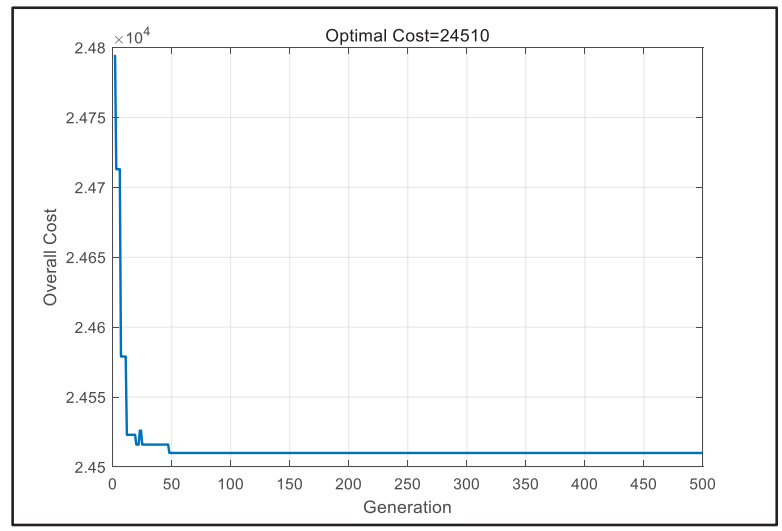

Fig 4. Recursive optimizing process of earthwork allocation.

\section{Conclusions and future work}

This paper develops an efficient earthwork allocation method based on a modified Genetic Algorithm with a self-adaptive mechanism mimicking the solution of threelayer multi-depot full-loaded Vehicle Route Problem. The research results are as follows:

- This method can minimize the total earthwork transportation cost by reducing the number of dispatched vehicles and global running shipment distance;

- The research result plays a guiding role in the problem of the earthwork allocation of the Winter
Olympic Skiing Course assisting the project in the initial design of the geomorphologic reshaping of mountains;

- This optimization method is full of potential to be improved due to its flexibility and compatibility, the easiness of modifying GA or combining GA with other heuristic algorithms enables larger room for improvement in the future. For instance, this paper only provides a twoway vehicle solution currently, a multi-vehicle solution is the goal that this research aims to finalize. Therefore, this optimization method can prolongate to any research field related to transportation and allocation with further studies.

\section{Acknowledgements}

This research was supported by National Key R\&D Program for the $13^{\text {th }}$-Five-Year Plan of China (2018YFF0300302 in 2018YFF0300300).

\section{References}

1. S. H. Kang, J. W. Seo and K. G. Baik, American Society of Civil Engineers Construction Research Congress 2009, 151-160 (2009).

2. M., Parente, P., Cortez, A., Gomes and Correia, (Pergamon Press, Inc., 2015).

3. G. B. Dantzig and J. H. Ramser, Management science 6 (1), 80-91 (1959).

4. J. Holland, Control and artificial intelligence (1975). 
5. R. Montemanni, L. M. Gambardella, A. E. Rizzoli and A. V. Donati, Journal of Combinatorial Optimization 10 (4), 327-343 (2005).

6. R. Eberhart and J. Kennedy, presented at the MHS'95. Proceedings of the Sixth International Symposium on Micro Machine and Human Science, 1995 (unpublished).
7. M. Dorigo, PhD Thesis, Politecnico di Milano (1992).

8. F. Glover, ORSA Journal on computing 1 (3), 190206 (1989).

9. S. Rui, Z. Li-hua, Z. Li-na and D. Bing-jie, Logistics Sci-Tech 39 (01), 39-43 (2016).

10. Floyd and R. W., Communications of the ACM 5 (6), 345 (1962). 